\title{
Brustzentren im Nationalen Krebsplan: Versorgung, Leitlinien und Dokumentation
}

\author{
M. W. Beckmann, M. P. Lux
}

Am 24. Februar 2010 wurde der Nationale Krebsplan vom Bundesministerium für Gesundheit (BMG) veröffentlicht [1]. Hintergrund des durch das BMG, die Deutsche Krebsgesellschaft e.V. (DKG), die Deutschen Krebshilfe e.V. (DKH) und die Arbeitsgemeinschaft Deutscher Tumorzentren e.V. (ADT) initiierten Planes ist die weiterhin hohe Krebsneuerkrankungsrate in Deutschland. Aufgrund der hohen Inzidenz von Krebserkrankungen der Mamma hat dieses Thema exemplarisch eine zentrale Rolle in der Versorgung von Frauen mit Krebserkrankungen und somit im Nationalen Krebsplan [2]. Für die in Deutschland etablierte Versorgung von Patientinnen mit einem Mammakarzinom in der zertifizierten Brustzentren nach den Kriterien der DKG und der Deutschen Gesellschaft für Senologie e.V. (DGS) hat der Nationale Krebsplan mehrere bedeutende Auswirkungen, welche im Folgenden erläutert werden.

\section{Weiterentwicklung der onkologischen Versorgungsstrukturen \\ $\nabla$}

Die zertifizierten Brustzentren sind flächendeckend etabliert (Behandlung von 91,5\% aller Mammakarzinompatientinnen in Deutschland, Stand 31.12.2010). Es stellt sich die Frage, in wie weit der Nationale Krebsplan für diese noch relevant sein kann.

Zunächst geht es um die Stärkung des Zentrumsbegriffes von gesundheitspolitischer Seite. Generell sind Zentrumsbezeichnungen, wie z.B. „Brustzentrum“, keine gesetzlich geschützten Begriffe. Mit der Definition des Zentrumsbegriffs durch den Nationalen Krebsplan erfolgt hier eine deutliche Stärkung. Dieses wird im Handlungsfeld 2 im Rahmen der Zielsetzung zur Weiterentwicklung der onkologischen Versorgungsstrukturen und Qualitätssicherung abgebildet, in dem es heißt, dass es sich bei Zentren um „ein Netz von qualifizierten und gemeinsam zertifizierten, interdisziplinären und transsektoralen, ggf. standortübergreifenden Einrichtungen, die möglichst die gesamte Versorgungskette für Betroffene abbilden" handelt. Zudem wird ein bundesweit einheitliches Konzept der Zertifizierung und Qualitätssicherung mit einheitlichen Bezeichnungen für onkologische Versorgungsstrukturen angestrebt. Es ist ein wichtiger Schritt, dass sich die Gesundheitspolitik mit dem Thema befasst. Dieses stärkt die zertifizierten Zentren und vermag in Zukunft einen Wildwuchs an selbst ernannten Zentren mit unklaren bzw. nicht geschützten Begriffen zu verhindern. Des Weiteren wird auch die Problematik der Finanzierung dieser Strukturen unterstützt. Es besteht die Hoffnung, dass die Verhandlungen für Zuschläge in Zukunft erleichtern werden.

\section{Leitliniengerechte Therapie}

$\nabla$

Eine fachliche Anforderung an zertifizierte Brustzentren ist die leitliniengrechte Therapie. Der Wert der Leitlinien wird weiter durch den Nationalen Krebsplan gestärkt. Eine vorhandene S3-
Leitlinie stellt für eine Tumorentität eine gewisse Planungssicherheit dar, so lange sie aktuell und in der Methodik korrekt durchgeführt worden ist. Das von der Arbeitsgemeinschaft der Wissenschaftlichen Medizinischen Fachgesellschaft (AWMF), der DKH und der DKG aufgelegte Onkologische Leitlinienprogramm (OL) dient der Erarbeitung von S3-Leitlinien für die verschiedensten Tumorentitäten. Ziel ist es hier, sowohl organspezifische (zum Beispiel Mamma oder Ovar) wie aber auch organübergreifende (Psychoonkologie, Supportivmaßnahmen, Palliativmedizin, Schmerztherapie und andere) Themenkomplexe zu definieren, um sowohl nachvollziehbare Versorgungsstandards zu implementieren, als auch bestehende Leitlinien weiterzuentwickeln, z.B. die S3-Leitlinie Diagnostik, Therapie und Nachsorge des Mammakarzinoms der Frau oder die S3-Leitlinie BrustkrebsFrüherkennung in Deutschland. Als obligate Bestandteile der Leitlinien müssen Qualitätsindikatoren definiert werden. Diese unterliegen einer Kosten-Nutzen- beziehungsweise Kosten-Effektivitätsanalyse.

Durch den Gesetzgeber unterstützte Leitlinien vermögen in Zukunft onkologische Therapieentscheidungen zu stärken, und die Angst vor Regressen zu reduzieren - betreffen also somit direkt den klinischen Alltag im zertifizierten Brustzentrum.

\section{Qualitätssicherung und Dokumentation im Nationalen Krebsplan $\nabla$}

Onkologische Zentrumsbildung mit Zertifizierung durch unabhängige externe Gutachter und Fachgesellschaften haben sowohl einen qualitätssichernden als auch qualitätsoptimierenden Effekt. Dieser kommt in Form einer verbesserten Prozess- und Ergebnisqualität den Patientinnen zugute. Für diesen Nachweis ist jedoch eine Messbarkeit der Parameter für die unterschiedlichen Dimensionen der Qualität erforderlich.

Insbesondere die zertifizierten Brustzentren, welche Patientinnen mit einem Mammakarzinom auf der höchsten Versorgungsstufe betreuen, benötigen zusätzliche personelle und materielle Ressourcen zur Erfüllung und Dokumentation der geforderten Qualitätsparameter, die jedoch aktuell nicht von den Kostenträgern erstattet werden.

Die klinischen Krebsregister sind eine wichtige Hilfe, insbesondere in der Erfassung der Ergebnisqualität, sind jedoch in Deutschland derzeit sehr unterschiedlich in der Follow-up-Quote und auch in der Unterstützung der zertifizierten Zentren, so dass diese häufig das Follow-up noch durch eigene Maßnahmen erheben müssen (z.B. Anschreiben und Anrufe der niedergelassenen Ärztinnen und Ärzte). Dieser Aspekt wurde nun im Rahmen des Nationalen Krebsplanes aufgegriffen. Im Handlungsfeld 2 fordert das Ziel 8 die aussagekräftige onkologische Qualitätsberichterstattung für die Leistungsbringer, die Entscheidungsträger und die Patientinnen und Patienten [Bundesministerium des Bundes, 2010]. Als erforderliches Instrument werden die klinischen Krebsregister benannt, welche in Kooperation mit den epidemio- 
logischen Krebsregistern tätig sein sollen. Mehrfachdokumentation, unterschiedliche Dokumentationssysteme und Teildokumentationen sind zukünftig nicht akzeptabel. In Bezug auf die Qualitätssicherung durch Dokumentation war es unstrittig, dass nur eine einmalige Dokumentation für einen horizontalen Langzeitverlauf einer Krebserkrankung erfolgen darf. Somit wird die flächendeckende Etablierung von klinischen Krebsregistern gefordert. Eine obligate Dokumentation sollte nach Umsetzung des im Juli 2009 verabschiedeten Bundeskrebsregistergesetzes möglich sein. Folgend sollen klinische Krebsregister bundesweit aufbzw. ausgebaut und die hierfür erforderlichen gesetzlichen Finanzierungsregelungen geschaffen werden.

Zu diesem Ziel hat das BMG eine Ausschreibung durchgeführt, um die Machbarkeit der Implementierung von klinischen Krebsregistern zu prüfen und eine Kostenanalyse zu erstellen. Das Gutachten wurde von Prognos im Oktober 2010 vorgestellt [3]. Um den Aufwand und Nutzen eines flächendeckenden Ausbaus klinischer Krebsregister abschätzen zu können, wurde eine Bestandsaufnahme der bestehenden klinischen Krebsregister durchgeführt und der Aufwand einer Umsetzung eines bundesweit flächendeckenden Systems im Vergleich zu möglichen Einsparpotenzialen abgeschätzt. Das Ergebnis der Kosten-Nutzen-Analyse fiel positiv aus, da ineffiziente Doppelstrukturen der Datenerfassung und Datenauswertung beseitigt und dadurch Synergieeffekte erreicht werden können [3].

Zudem sind bei einem bundesweit einheitlichen Konzept klinischer Krebsregistrierung im Sinne von Ziel 8 positive Effekte hinsichtlich der Behandlungsqualität zu erwarten.

Vor dem Hintergrund der im Rahmen des Nationalen Krebsplans formulierten Ziele zeigt sich jedoch, dass zum gegenwärtigen Zeitpunkt keines der Register die Anforderungen vollends erfüllen kann.

Als Fazit empfiehlt das Gutachten mehrere Handlungsstrategien, u.a. die Herstellung einer gesetzlichen Verbindlichkeit mit bundesweit einheitlichen Vorgaben, Definition des Aufgabenprofils und Standards gemeinsam mit Datennutzern, z. B. den zertifizierten Brustzentren. Dies hat zum Ziel, eine Vergleichbarkeit und Kompatibilität der Daten der klinischen Krebsregister untereinander und mit epidemiologischen Registern zu gewährleisten und eine Harmonisierung der Dokumentationsprogramme zu erreichen, eine Mehrfachdokumentation zu vermeiden, den Meldeweg über die klinischen Krebsregister festzuschreiben und zur Kooperation mit den Organkrebszentren zu verpflichten, da hierdurch erhebliche Synergieeffekte erzielt werden können. Nicht zuletzt fordert das Gutachten die erforderliche Sicherstellung der Finanzierung, u. a. auch über Mittel der Krankenkassen. Somit kann sowohl der Nationale Krebsplan als auch das Gutachten von Prognos weitereichende positive Folgen für zertifizierte Brustzentren haben.

\section{Fazit \\ $\nabla$}

Zusammenfassend lässt sich in Bezug auf die zertifizierten Brustzentren festhalten, dass der Nationale Krebsplan eine große Chance darstellt, die etablierten Versorgungsstrukturen zu stärken. Über adäquate Dokumentationserstattung kann eine hochwertige Dokumentations- und Datenqualität gewährleistet werden, die einerseits den Verlauf der Erkrankung nicht nur im Hinblick auf Tod, aber insbesondere auch auf die Parameter Rezidiv und Metastasen ermöglicht, andererseits die zertifizierten Brustzentren entlastet, welche einen erheblichen personellen Aufwand betreiben, um die in den Erhebungsbögen geforderten Kennzahlen nachzuweisen.

\section{Literatur}

1 Bundesministerium des Bundes. Nationaler Krebsplan. Stand: Februar 2010. http://www.bmg.bund.de/cln_169/nn_1168248/SharedDocs/ Standardartikel/DE/AZ/N/Glossarbegriff-Nationaler-Krebsplan.html\# doc1632822bodyText14

2 Beckmann MW. Nationaler Krebsplan des Bundesministeriums für Gesundheit - Strategieplan der Deutschen Krebsgesellschaft. Frauenheilkunde up2date 2009; 5: 323-329

3 Blatt $K$, Hölterhoff $M$, Henkel $M$ et al. Aufwand-Nutzen-Abschätzung zum Ausbau und Betrieb bundesweit flächendeckender klinischer Krebsregister. Prognos. http://www.bmg.bund.de/fileadmin/redaktion/ pdf_publikationen/Gutachten-Aufwand-Nutzen-Abschaetzung-Krebsregister.pdf 2010

\section{Bibliografie}

DOI http://dx.doi.org/10.1055/s-0031-1271513

Senologie 2011; 8: 97-98

(c) Georg Thieme Verlag KG Stuttgart · New York · ISSN 1611-6453

\section{Korrespondenzadresse}

Prof. Dr. med. Matthias W. Beckmann

Universitätsklinikum Erlangen

Ärztlicher Direktor der Frauenklinik

Universitätsstr. 21-23

91054 Erlangen

Matthias.Beckmann@uk-erlangen.de 